\title{
Negative four-terminal resistance as a probe of crossed Andreev reflection
}

\author{
D. Beckmann* \\ Forschungszentrum Karlsruhe, Institut für Nanotechnologie, P.O. Box 3640, D-76021 Karlsruhe, Germany \\ H. v. Löhneysen \\ Forschungszentrum Karlsruhe, Institut für Festkörperphysik, \\ P.O. Box 3640, D-76021 Karlsruhe, Germany, and Physikalisches Institut, \\ Universität Karlsruhe, D-76128 Karlsruhe, Germany
}

(Dated: September 30, 2018)

\begin{abstract}
We report on the experimental investigation of electronic transport in superconductor-ferromagnet spin-valve structures. Our samples consist of two ferromagnetic iron leads forming planar tunnel contacts to a superconducting aluminum wire. At energies below the superconducting gap, we observe a negative four-probe resistance that can be explained by crossed Andreev reflection.
\end{abstract}

Quantum mechanics predicts a non-local correlation of spatially separated particles that is stronger than that allowed by classical theory. This correlation, known as entanglement, has been tested experimentally on pairs of photons ${ }^{1}$ and massive particles ${ }^{2}$, see also ${ }^{3}$. An intriguing question is whether a stream of spatially separated, entangled electron pairs can be created and manipulated in a solid-state environment, with possible future applications in quantum information processing. A promising implementation of solid-state entanglers are heterostructures involving a superconductor,$\frac{4.5}{,}$ as in a superconductor the electrons come naturally in the form of Cooper pairs, which are in an entangled singlet state. The challenge is to create structures where the two electrons of a Cooper pair can be spatially separated without destroying entanglement. If two normal-metal contacts are attached to a superconductor at a distance smaller than the coherence length of the superconductor, it has been predicted that an electron injected into the superconductor may be transmitted into the second contact as a hole, thereby creating a Cooper pair in the superconductor, and leaving behind two entangled holes in the two normal metal contacts 6.7 . There is a competition between this process, called crossed Andreev reflection (CAR), and elastic cotunneling (EC), where the incident electron gets transmitted to the second contact via a virtual state in the superconductor. Quantitative understanding of the contributions of CAR and competing processes like $\mathrm{EC}$ is a prerequisite to building a solid-state entangler based on superconductor hybrid structures.

Crossed Andreev reflection has been studied experimentally in superconductor/ferromagnet ${ }^{8}$ and superconductor/normal-metal ${ }^{9}$ structures. In a recent experiment ${ }^{8}$ on transport properties of superconductor/ferromagnet spin-valve structures, we have observed spin-dependent transport in the superconductor at energies much smaller than the superconducting gap. The observed signal decayed on the length scale of the coherence length of the superconductor, and could be explained by theoretical predictions for the superposition of crossed Andreev reflection and elastic cotunneling. Here, we present an experimental study that allows us to discriminate the two processes, and also rule out

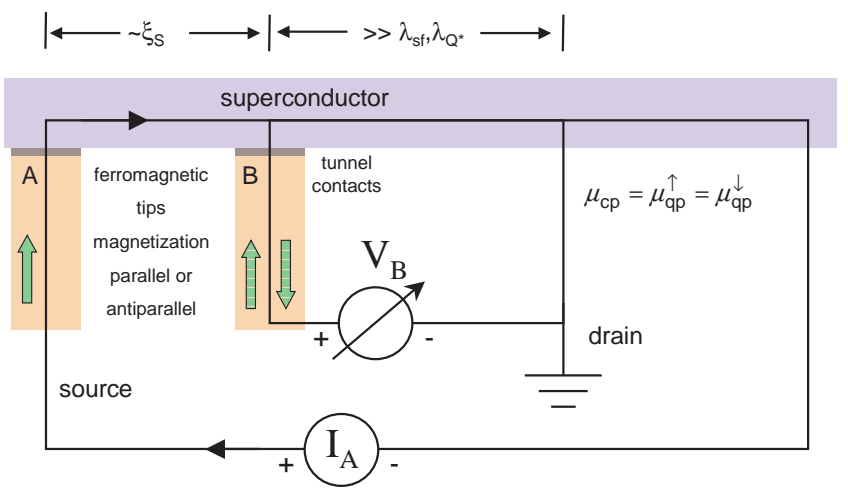

FIG. 1: (Color online) Schematic view of the experiment. Two ferromagnetic leads form tunnel contacts to a superconducting bar. Contact $\mathrm{A}$ is used to inject a current, and the voltage at contact $B$ is measured referenced to the drain voltage probe. The magnetization alignment of the ferromagnets is either parallel or antiparallel.

sequential tunneling or non-equilibrium effects as a possible cause of the observed signals.

The schematic view of the experiment is shown in Fig. 11 Two ferromagnetic wires A and B are attached via tunnel contacts to a superconducting bar. Contact A (source) is used to inject a current into the superconductor, which then flows along the bar. The (Ohmic) drain contact, which lies inside the current path, is used as a reference of the chemical potential of the superconductor. Contact B is used to measure voltage relative to the drain contact. There are several possible mechanisms for the observation of a finite voltage at contact $B$ despite the fact that voltage is measured across a superconductor: EC (CAR) emits an electron (hole) from the superconductor into contact $\mathrm{B}$ for an electron injected into contact A, thereby leading to a voltage that has equal (opposite) sign compared to the injector voltage. Both processes are elastic and coherent, and involve virtual quasiparticle states in the superconductor, i.e. they may occur at energies below the superconducting energy gap, and over the length scale of the coherence length $\xi_{\mathrm{S}}$ of the superconductor. Note that for CAR, the chemical potential of 


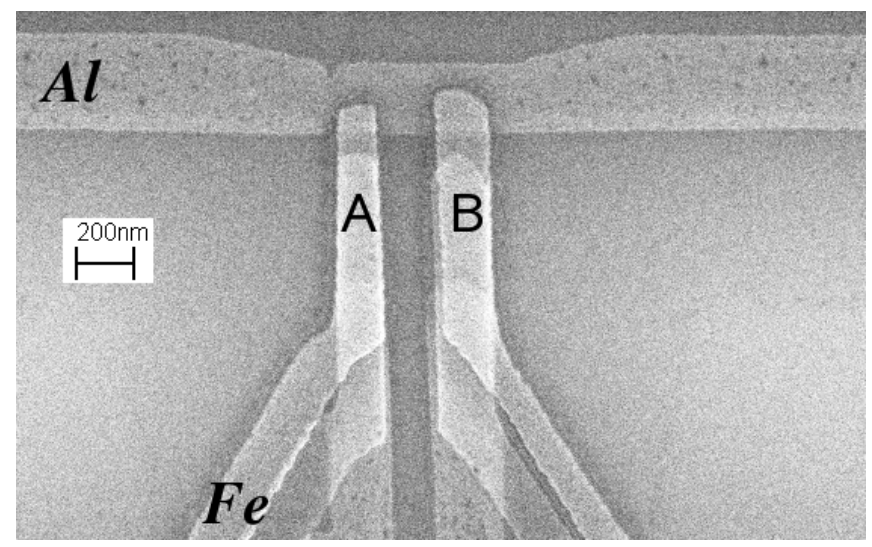

FIG. 2: SEM image of the two contacts of sample II.

the detector contact $\mathrm{B}$ may lie outside the voltage window spanned by source and drain contact, which has been predicted previously for a slightly different setup 10

In addition to coherent subgap processes, an electron may tunnel into an allowed state in the superconductor and sequentially tunnel out at contact B. Sequential tunneling, like elastic cotunneling, yields a voltage $V_{\mathrm{B}}$ of the same sign as $V_{\mathrm{A}}$. Sequential tunneling requires an electron to have enough energy to overcome the spectral gap, which can be supplied by the applied voltage, thermal excitation or external noise. Alternatively, the gap may be smeared out by quasi-particle life-time broadening, the inverse proximity effect in the contact regions, or the magnetic stray fields of the electrodes. Sequential tunneling may be either elastic and coherent, or incoherent, generating a non-equilibrium charge $\underline{11.12}$ and $\operatorname{spin}^{13}$ accumulation in the superconductor. It is known that non-equilibrium quasiparticle populations in superconductors relax only slowly due to electron-phonon scattering, 14 and accordingly the decay length of the nonequilibrium can be quite large. From the data of our previous experiment, $\stackrel{8}{=}$ we obtain the charge imbalance relaxation length $\lambda_{\mathrm{Q}^{*}} \approx 3 \mu \mathrm{m}$ and the normal-state spin diffusion length $\lambda_{\mathrm{sf}} \approx 1 \mu \mathrm{m}$. A $10 \mu \mathrm{m}$ distance between source and drain contact was chosen, to ensure that any non-equilibrium quasi-particle populations which may be injected at contact $\mathrm{A}$ have relaxed at the drain reference contact. Therefore, the chemical potentials of both quasiparticle spin species $\left(\mu_{\mathrm{qP}}^{\uparrow, \downarrow}\right)$ and the Cooper pairs $\mu_{\mathrm{cp}}$ are the same, and the drain contact can be considered as a faithful measure of the equilibrium chemical potential of the superconductor.

The experiments were performed on mesoscopic spinvalve structures made of ferromagnetic iron and superconducting aluminum by means of e-beam lithography and shadow evaporation. The contact region of sample II is shown in the SEM image in Fig. 22 In a first evaporation step, $15 \mathrm{~nm}$ of iron is evaporated from a direction almost normal to the plane of an oxidized silicon wafer. The purpose of this first iron layer is only to ensure Ohmic contacts everywhere in the structure except
$\mathrm{T}(\mathrm{K})$

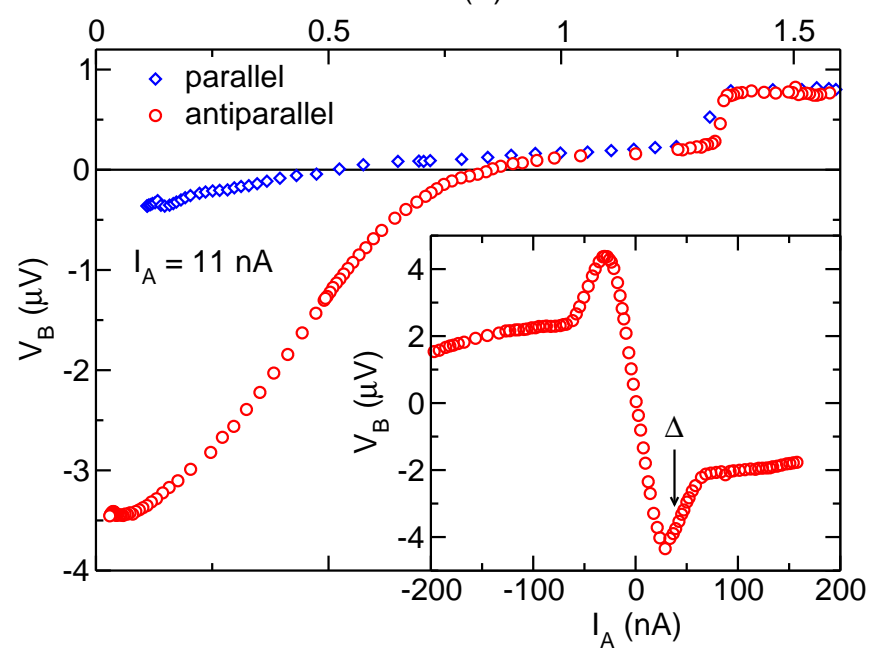

FIG. 3: (Color online) Temperature dependence of the detector voltage $V_{\mathrm{B}}$ at fixed injector current $I_{\mathrm{A}}=11 \mathrm{nA}$ for parallel (diamonds) and antiparallel (circles) magnetization alignment for sample II. Inset: $V_{\mathrm{B}}$ as a function of $I_{\mathrm{A}}$ in the antiparallel alignment at $T=25 \mathrm{mK} . \Delta$ marks the current that corresponds to $V_{\mathrm{A}}=200 \mu \mathrm{V}$, i.e. the gap energy of aluminum.

for the two tunnel contacts A and B. Then, $25 \mathrm{~nm}$ of aluminum are evaporated under an oblique angle, forming the aluminum wire of $200 \mathrm{~nm}$ width seen in the upper part of the SEM image. The aluminum is then oxidized in situ admitting a pressure of $60 \mathrm{~Pa}$ of pure oxygen for about $1 \mathrm{~min}$. Subsequently, a second layer of $20 \mathrm{~nm}$ of iron is evaporated from the opposite direction to form the two tunnel contacts A and B. After lift-off, the samples were bonded and mounted into a shielded box thermally anchored to the mixing chamber of a dilution refrigerator. All wire connections from the top of the cryostat to the sample chamber were filtered through discrete RC low-pass filters, lossy coaxial lines and a copper powder filter to eliminate noise and high-frequency thermal photons from the sample chamber. The experiments presented here were carried out using a battery and a resistor network as DC current source, and a nanovoltmeter for voltage measurements. The low-bias results were confirmed using an $\mathrm{AC}$ resistance bridge. Here, we present data from three samples I, II and III.

Figure 3 shows the detector voltage $V_{\mathrm{B}}$ as a function of temperature for sample II. Here, the magnetization of injector and detector contact have been aligned parallel or antiparallel at $T>T_{\mathrm{c}}$ by monitoring the normal-state spin-valve signal, and then subsequently the sample is cooled down at zero magnetic field. Above $T_{\mathrm{c}}$, the observed voltage is essentially due to the Ohmic resistance of the $10 \mu \mathrm{m}$ long aluminum strip between contact B and the drain reference contact, plus the small spin accumulation signal. At $T_{\mathrm{c}} \approx 1.35 \mathrm{~K}$, the Ohmic resistance disappears, and a small positive signal remains around $1 \mathrm{~K}$. At lower temperature, the detector voltage becomes 
negative for both magnetization alignments, but with a much larger magnitude for antiparallel alignment.

The dependence of the detector voltage $V_{\mathrm{B}}$ on bias current $I_{\mathrm{A}}$ is shown in the inset of Fig. [3] At low bias current $\left|I_{\mathrm{A}}\right|<30 \mathrm{nA}$, the slope is negative, turning into a positive slope at higher bias. By comparison with the local current-voltage relation (IV) of the injector contact we see that the slope reversal corresponds to $V_{\mathrm{A}} \approx 200 \mu \mathrm{V}$, i.e. the superconducting energy gap of aluminum. Thus, we conclude that the positive slope corresponds to the onset of the transmission of quasiparticles through allowed states above the gap.

From the measured non-local voltage as a function of current, the non-local conductance can be calculated by using the conductance matrix

$$
\left(\begin{array}{c}
I_{\mathrm{A}} \\
I_{\mathrm{B}}
\end{array}\right)=\left(\begin{array}{ll}
G_{\mathrm{A}} & G_{\mathrm{X}} \\
G_{\mathrm{X}} & G_{\mathrm{B}}
\end{array}\right)\left(\begin{array}{c}
V_{\mathrm{A}} \\
V_{\mathrm{B}}
\end{array}\right),
$$

which contains the local conductances $G_{\mathrm{A}, \mathrm{B}}$ of the injector (A) and detector (B) contact, and the non-local conductance $G_{\mathrm{X}}$. For voltage detection at B, we have $I_{\mathrm{B}}=0$, and the current due to the non-local conductance, $I_{\mathrm{X}}=G_{\mathrm{X}} V_{\mathrm{A}}$, is canceled by the backflow due to the local conductance, $G_{\mathrm{B}} V_{\mathrm{B}}$. By balancing the total currents, we assume that all charge carriers emitted into contact $\mathrm{B}$ by non-local processes relax to the equilibrium chemical potential of wire $B$ before they tunnel back. In that case, as $V_{\mathrm{B}}$ never exceeds a few microvolts, we can replace $G_{\mathrm{B}}$ by its low-bias value, and the energy dependence of $V_{\mathrm{B}}$ is given only by the dependence of $G_{\mathrm{X}}$ on $V_{\mathrm{A}}$. With $G_{\mathrm{X}} \ll G_{\mathrm{A}}$, we find

$$
G_{\mathrm{X}}=\frac{d I_{\mathrm{B}}}{d V_{\mathrm{A}}}=-G_{\mathrm{B}} \frac{d V_{\mathrm{B}}}{d V_{\mathrm{A}}} .
$$

The resulting non-local differential conductance $d I_{\mathrm{B}} / d V_{\mathrm{A}}$ as a function of injector voltage $V_{\mathrm{A}}$, together with the local differential conductances of injector and detector contact measured directly, is shown in Fig. 4

The local conductance of the detector exhibits a suppression at subgap energies, and peaks at the energy of the gap, $\Delta \approx 200 \mu \mathrm{eV}$, which at least qualitatively corresponds to the expected BCS tunneling characteristics. For the injector, neither the subgap suppression nor the peaks are well resolved. For both injector and detector, the subgap conductance is much too large to be compatible with a simple BCS behavior, and has a downward curvature at low bias, which is not expected for either BCS quasiparticle tunneling, or Andreev reflection. A strong enhancement of Andreev reflection at low bias in mesoscopic NS tunnel junctions is known to occur due to the constructive interference of time-reversed electron and hole trajectories on the normal metal side of the tunnel junction (reflectionless tunneling) 15 While reflectionless tunneling exhibits the decrease of conductance with increasing bias in the subgap regime that we observe, it should not occur in our structures, as time-reversal sym-

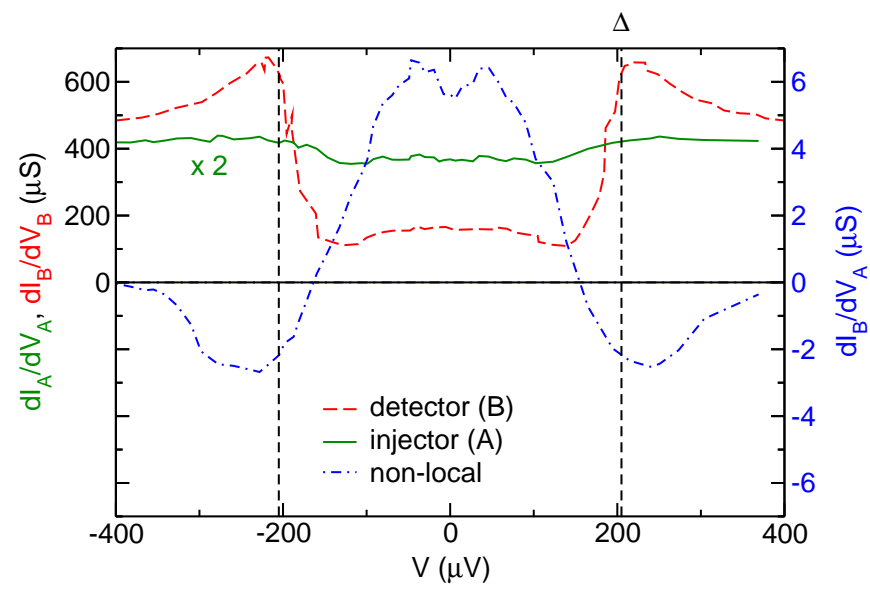

FIG. 4: (Color online) Local differential conductances $d I_{\mathrm{A}} / d V_{\mathrm{A}}$ and $d I_{\mathrm{B}} / d V_{\mathrm{B}}$ (left scale), and non-local differential conductance $d I_{\mathrm{B}} / d V_{\mathrm{A}}$ (right scale), of sample II at $T \approx$ $25 \mathrm{mK} . d I_{\mathrm{A}} / d V_{\mathrm{A}}$ is doubled for clarity.

metry is broken in the ferromagnetic electrodes. However, it has been predicted ${ }^{16}$ that an interference enhancement of Andreev reflection also occurs on the superconducting side of the interface in restricted geometries. The formalism used to describe this enhancement is the same as used later to describe crossed Andreev reflection in diffusive metals 17.18

The non-local conductance is positive (corresponding to crossed Andreev reflection) in the subgap regime, and becomes negative (corresponding to electron transmission) as the injector voltage approaches the superconducting gap. At the gap, a negative peak is observed, mimicking the BCS density of states peak seen in the local conductance. The overall magnitude of the nonlocal conductance is about a factor of 100 smaller than the local conductance. Using the model of twodimensional diffusion ${ }^{16.18}$, we find that a relative reduction of the non-local conductance compared to the local Andreev conductance of two orders of magnitude is realistic. However, using the full quantitative expression ${ }^{16}$, the local Andreev conductance should be of the order of $G_{\mathrm{N}}^{2} R_{\square} \approx 1 \mu \mathrm{S}$, where $G_{\mathrm{N}}$ is the normal-state tunnel conductance, and $R_{\square}$ the normal-state sheet resistance of the superconductor. This estimate is much less than the conductances actually observed. We suspect that pinholes in the oxide barrier lead to an enhanced Andreev conductance.

Figure [5] shows the non-local conductance as a function of injector voltage for three different samples in the antiparallel magnetization state. For samples I and II, the data are qualitatively the same, with a dominating positive signal at low bias, and a negative peak near the energy gap at about $200 \mu \mathrm{V}$. However, sample III shows a different behavior. The non-local conductance is negative at small bias, has a positive peak at about $80 \mu \mathrm{V}$, and then decays without any clear feature near the gap. For sample III, the detector voltage $V_{\mathrm{B}}$ has the same sign 
TABLE I: Characteristic parameters of our samples. Average transmission probability $t$ of the tunnel contacts, elastic mean free path $l_{\mathrm{el}}$, coherence length $\xi$, normalized contact distance $d / \xi$, and inverse diffusion time $\hbar / \tau_{\mathrm{D}}$.

\begin{tabular}{lccccc}
\hline \hline sample & $t$ & $\begin{array}{c}l_{\mathrm{el}} \\
(\mathrm{nm})\end{array}$ & $\begin{array}{c}\xi \\
(\mathrm{nm})\end{array}$ & $d / \xi$ & $\begin{array}{c}\hbar / \tau_{\mathrm{D}} \\
(\mu \mathrm{eV})\end{array}$ \\
\hline I & $8 \times 10^{-5}$ & 13 & 140 & 1.3 & 120 \\
II & $4 \times 10^{-5}$ & 10 & 120 & 1.7 & 70 \\
III & $2 \times 10^{-5}$ & 5 & 85 & 2.3 & 40 \\
\hline \hline
\end{tabular}

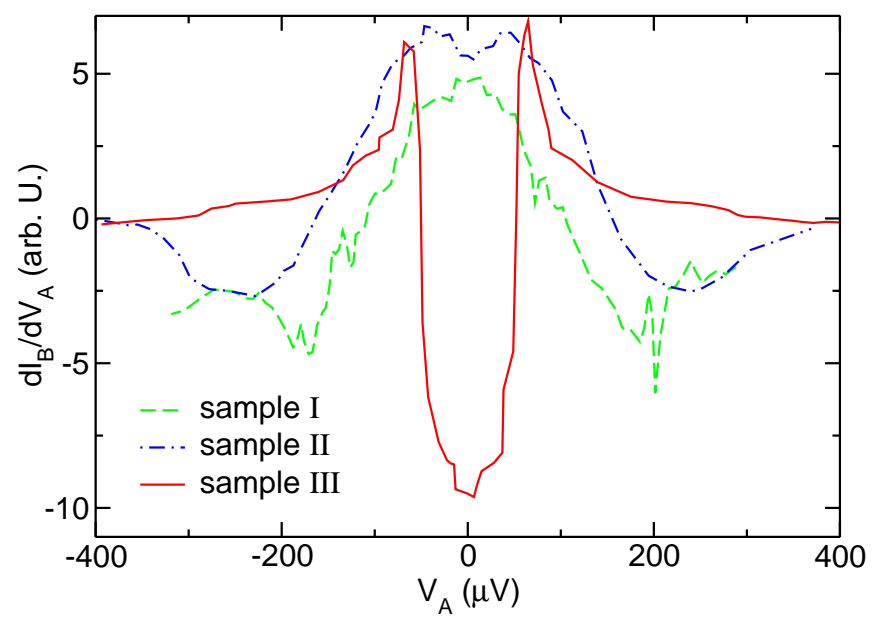

FIG. 5: (Color online) Non-local differential conductance $d I_{\mathrm{B}} / d V_{\mathrm{A}}$ as a function of injector voltage $V_{\mathrm{A}}$ for three different samples. The data are scaled to a similar amplitude.

as the injector voltage over the whole IV trace.

The theoretical predictions for the non-local conductance due to CAR and EC in the tunneling limit $\underline{19}$ show that both contributions are of the same magnitude and opposite sign. Therefore, without spin selection, the nonlocal subgap conductance is expected to be zero. Including spin selection a negative (positive) conductance of equal magnitude is expected for parallel (antiparallel) alignment. For increasing contact transparency, EC is predicted to give a larger absolute contribution than $\mathrm{CAR}^{20}$ Here, we observe a dominantly positive non-local conductance for samples I and II, and a negative signal for sample III. In Table \we summarize the experimental parameters of the three samples. As can be seen, the average contact transparency estimated from the normal-state tunnel resistance systematically decreases from sample I to III. A transition from dominating CAR to dominating EC with decreasing transparency is inconsistent with the predictions of Ref. 20. The elastic mean free path $l_{\mathrm{el}}$ of the aluminum also systematically decreases from sample I to III, and thereby the coherence length $\xi$ decreases and the normalized contact dis- tance $d / \xi$ increases. The dependence of CAR and EC on contact distance is expected to be the same 17.19 which means that except for an overall signal decrease, no qualitative change is expected with increasing $d / \xi$. Recently, it has been predicted ${ }^{21}$ that coupling to the electromagnetic environment (dynamical Coulomb blockade) may discriminate CAR and EC, and also explain a transition between dominating CAR and $\mathrm{EC}$ at a finite bias voltage related to typical energies of environmental modes, either symmetric or antisymmetric with respect to the two contacts. As the elastic mean free path of the aluminum may affect the environmental modes, we speculate that the qualitatively different behavior of sample III compared to I and II may be related to the decrease in $l_{\mathrm{el}}$, probably in conjunction with the decrease in contact transparency.

The non-local conductance of sample III is qualitatively consistent with the observations made by Russo et $a^{.} \underline{\underline{9}}$ in a coplanar geometry, where a superconducting layer was sandwiched between two non-magnetic contacts. Russo et al. report a positive differential voltage (corresponding to a negative differential conductance) at low bias, which reverses sign at an energy scale well below the superconducting gap, and decays towards higher energies. The energy scale of the sign reversal was found to be the Thouless energy $E_{\mathrm{Th}}=\hbar / \tau_{\mathrm{D}}$, where $\tau_{\mathrm{D}}$ is the diffusion time corresponding to the thickness of the superconducting film (which coincides with the distance between the two normal metal contacts in a coplanar geometry). For comparison, we have calculated the energy scale $\hbar / \tau_{\mathrm{D}}$ corresponding to the diffusion time from contact $\mathrm{A}$ to $\mathrm{B}$ in our samples, and show the figures in table [1. Even though the sign reversal of the non-local conductance of sample III may be related to $\hbar / \tau_{\mathrm{D}}$, no clear features are seen at this energy for sample A and B.

To conclude, we have presented non-local voltage measurements on superconductor-ferromagnet spin-valve structures, and observed a negative four-probe resistance that provides unambiguous evidence for crossed Andreev reflection as the dominating subgap transport process in some samples. This is an important prerequisite for the use of crossed Andreev reflection in efficient entanglers. The qualitatively different behavior reported by Russo et al. and also observed in one of our samples requires further systematic experimental investigation as well as theoretical work.

We thank W. Belzig, D. Feinberg, R. Mélin and A. Levy-Yeyati for useful discussions, and especially P. Samuelsson, D. Sanchez, R. Lopez, E. Sukhorukov and M. Büttiker for bringing the source-drain window argument to our attention. This work was partly supported by the Deutsche Forschungsgemeinschaft within the Center for Functional Nanostructures. 
* e-mail address: detlef.beckmann@int.fzk.de

1 A. Aspect, J. Dalibard, and G. Roger, Phys. Rev. Lett. 49, 1804 (1982).

2 M. A. Rowe, D. Kielpinski, V. Meyer, C. A. Sackett, W. M. Itano, C. Monroe, and D. J. Wineland, Nature 309, 791 (2001).

3 M. Genovese, Physics Reports 413, 413 (2005).

${ }^{4}$ P. Recher, E. V. Sukhorukov, and D. Loss, Phys. Rev. B 63, 165314 (2001).

${ }^{5}$ G. Lesovik, T. Martin, and G. Blatter, Eur. Phys. J. B 24, 287 (2001).

6 J. M. Byers and M. E. Flatté, Phys. Rev. Lett. 74, 306 (1995).

7 G. Deutscher and D. Feinberg, Appl. Phys. Lett. 76, 487 (2000).

8 D. Beckmann, H. B. Weber, and H. v. Löhneysen, Phys. Rev. Lett. 93, 197003 (2004).

9 S. Russo, M. Kroug, T. M. Klapwijk, and A. F. Morpurgo, Phys. Rev. Lett. 95, 027002 (2005).
${ }^{10}$ F. J. Jedema, B. J. van Wees, B. H. Hoving, A. T. Filip, and T. M. Klapwijk, Phys. Rev. B 60, 16549 (1999).

11 J. Clarke, Phys. Rev. Lett. 28, 1363 (1972).

12 M. Tinkham and J. Clarke, Phys. Rev. Lett. 28, 1366 (1972).

13 M. Johnson, Appl. Phys. Lett. 65, 1460 (1994).

14 C. C. Chi and J. Clarke, Phys. Rev. B 19, 4495 (1979).

15 B. J. van Wees, P. de Vries, P. Magnée, and T. M. Klapwijk, Phys. Rev. Lett. 69, 510 (1992).

${ }^{16}$ F. W. J. Hekking and Y. V. Nazarov, Phys. Rev. B 49, 6847 (1994).

17 D. Feinberg, Eur. Phys. J. B 36, 419 (2003).

18 G. Bignon, M. Houzet, F. Pistolesi, and F. W. J. Hekking, Europhys. Lett. 67, 110 (2004).

19 G. Falci, D. Feinberg, and F. W. J. Hekking, Europhys. Lett. 54, 255 (2001).

20 R. Mélin and D. Feinberg, Phys. Rev. B 70, 174509 (2004).

21 A. Levy Yeyati, unpublished. 